\title{
Non-affine deformation of inherent structure as static signature of cooperativity in supercooled liquids
}

\author{
Emanuela Del Gado, Patrick Ilg, Martin Kröger and Hans Christian Öttinger \\ ETH Zürich, Polymer Physics, CH-8093 Zürich, Switzerland
}

(Dated: November 3, 2018)

\begin{abstract}
We unveil the existence of non-affinely rearranging regions in the inherent structures (IS) of supercooled liquids by numerical simulations of two- and three-dimensional model glass formers subject to static shear deformations combined with local energy minimizations. In the liquid state IS, we find a broad distribution of rather large rearrangements which are correlated only over small distances. At low temperatures, the onset of the cooperative dynamics corresponds to much smaller displacements correlated over larger distances. This finding indicates the presence of nonaffinely rearranging domains of relevant size in the IS deformation, which can be seen as the static counterpart of the cooperatively rearranging regions in the dynamics. This idea provides new insight into possible structural signatures of slow cooperative dynamics of supercooled liquids and supports the connections with elastic heterogeneities found in amorphous solids.
\end{abstract}

PACS numbers: 61.43.Fs,64.70.Q-,05.20.Jj

When a liquid is cooled down to its glass transition temperature, particle motion slows down enormously and becomes highly cooperative [1]: The more the system becomes glassy, the more the relaxation requires cooperative rearrangements of a large number of particles. Experimental and theoretical investigations as well as simulation results on dynamical heterogeneities strongly support the presence of cooperatively rearranging regions of growing size [1, 2, 3, 4]. However, it is still debated whether and how the onset of such cooperative dynamics can take place without any apparent or straightforward connection to structural changes. Such links have been searched for in various directions. For example, different connections between the local propensity of particles to motions and the underlying structural features of the system have been investigated, but they still remain quite elusive [5].

An alternative (and so far complementary) description of the dynamics of supercooled liquids is based on the way the system explores its potential energy surface [6]. When the system enters the supercooled regime, the presence of local minima, or inherent structures (IS), turns out to be extremely important and to produce complex trajectories in the potential energy landscape: The time evolution of the system samples the basins associated to the local minima, or groups of such basins, on a relatively short time scale, whereas transitions between different groups of basins separated by major energy barriers take a much longer time and imply a slow, aging process, during which the system is out-of-equilibrium. Such a description, however, does not fully account for the cooperative dynamics associated to dynamical heterogeneities. Yet, possible connections have been discussed [6] and recent works have gathered additional insights [7, 8, 9, 10, 11, 12].

In the present paper, we report about a signature of the onset of cooperative dynamics in the structural features of supercooled liquids from a novel perspective. The approach is motivated by a recent theory [13] - based on a general framework of nonequilibrium thermodynamics which relates the response of a glassy system to an applied deformation to the corresponding change of its IS. In contrast to previous approaches, here it is suggested that the reversible part of the dynamics changes considerably when approaching the glass transition. Since the reversible dynamics basically mirrors the space transformation behavior of the system, the suggestion in 13. guides the way toward investigations of static rather than dynamical properties. Accordingly, the most important feature of IS transformation is a hindrance to affine deformation: The typical linear size of the regions where non-affine deformations take place is roughly the particle size in the liquid and is expected to become larger upon approaching the glass transition.

We explore these theoretical considerations by computer simulations of different model systems for supercooled liquids subject to static shear deformations with small amplitudes. In the liquid phase, a broad distribution of rearrangements is found, centered around a rather large mean value. When the system enters the supercooled regime, instead, its IS displays the onset of an enhanced collective behavior with much smaller displacements. This feature, which does not depend on the particular model or deformation considered, has been detected here for the first time and indicates the existence of non-affinely rearranging regions of relevant size in the IS of supercooled liquids. The presence of such collective rearrangements in the IS response is strongly evocative of the cooperativity characterizing the dynamics, and it is in fact observed in the same range of temperatures. On this basis, we propose that these non-affinely rearranging regions are the IS counterpart of the cooperatively rearranging regions observed so far only in the dynamics.

Methods and numerical simulations. We consider three well established model systems for supercooled liquids: (a) The three-dimensional (3D) binary Lennard-Jones mixture of Ref. [14]; (b) The 3D soft spheres binary mixture studied in Ref. [15]; (c) The 2D soft spheres binary 
mixture in Ref. [16]. In all three models, the densities have been chosen such that crystallization is prevented. Upon cooling, these systems therefore go from the liquid to the supercooled and glassy regime. In order to investigate the response of IS to an applied deformation we have designed the following procedure [13]. First, starting from a particle configuration $X=\left\{\mathbf{r}_{i}\right\}$ equilibrated at a given temperature $T$, we deform it affinely $\mathbf{r}_{i} \rightarrow \mathbf{r}_{i}^{\mathrm{d}}$ and subsequently find the inherent structure of the deformed configuration $X^{\mathrm{dq}}=\left\{\mathbf{r}_{i}^{\mathrm{dq}}\right\}$. For the energy minimization we use a conjugate gradient algorithm. Second, starting from the same initial configuration $X$, we first find the IS corresponding to the initial configuration $X^{\mathrm{q}}=\left\{\mathbf{r}_{i}^{\mathrm{q}}\right\}$ and subsequently apply the same affine deformation to obtain $X^{\mathrm{qd}}=\left\{\mathbf{r}_{i}^{\mathrm{qd}}\right\}$. All subsequent analysis is based on the comparison between the two configurations $X^{\mathrm{dq}}$ and $X^{\mathrm{qd}}$, which is quantified by the mismatch, i.e. the nonaffine displacement field $\left\{\mathbf{d}_{i}=\mathbf{r}_{i}^{\mathrm{dq}}-\mathbf{r}_{i}^{\mathrm{qd}}\right\}$. In the limit of a flat energy landscape, $\left\{\mathbf{d}_{i}\right\}$ is identically zero. By lowering the temperature into the supercooled regime, we expect the presence of basins and barriers in the energy landscape to introduce collective contributions to the displacement field. We carefully chose the deformation amplitudes sufficiently large to make the system typically leave a basin. Supplementary information is given in the EPAPS document [17]. We have considered two different kinds of deformation: (i) The spatially modulated shear deformation $\mathbf{r}_{i} \rightarrow \mathbf{r}_{i}^{\mathrm{d}}=\mathbf{r}_{i}+\gamma \sin \left(k_{n} y_{i}\right) \mathbf{e}_{x}$, where $\gamma$ denotes the maximum amplitude of the deformation and $k_{n}=n \pi / L$ the wave-vector with $L$ the size of the simulation box; (ii) The homogeneous shear $\mathbf{r}_{i} \rightarrow \mathbf{r}_{i}^{\mathrm{d}}=\mathbf{r}_{i}+\gamma y_{i} \mathbf{e}_{x}$, where Lees-Edwards boundary conditions are used. We have applied the deformations $(i)$ and $(i i)$ to the systems $(a),(b)$ and $(c)$ with $\gamma$ varying between $10^{-4}$ and $10^{\circ}$. All quantities are given in reduced Lennard-Jones units. For all the systems we have prepared 20 to 50 independent samples of $N$ particles ( $N$ varying from 500 to 8000) [17] which have been carefully equilibrated [18]. All quantities discussed are averaged over the independently prepared samples and the error bars obtained from sample-to-sample fluctuations. Some simulations were performed using the LAMMPS code [19]. The following analysis concerns temperatures $T$ in the range from the high $T$ Arrhenius dependence of dynamic quantities to the onset of caging and cooperative dynamics.

Results. A first, qualitative understanding can be obtained via a simple visualization of the mismatch field $\left\{\mathbf{d}_{i}\right\}$ at different temperatures and for different amplitudes of deformation. In Fig. 1, $\left\{\mathbf{d}_{i}\right\}$ vectors are plotted for the system $(c)$ and deformation $(i i)$ at high temperature $T=1.0$ (left) and low temperature $T=0.46$ (right). The length of the vectors are suitably enlarged for clarity. At high $T$, for a fixed amplitude of deformation, the mismatch vectors display a relatively large magnitude as well as uncorrelated directions. This indicates that, at these temperatures, the local changes to the IS induced by our procedure are in fact dominated by the thermal
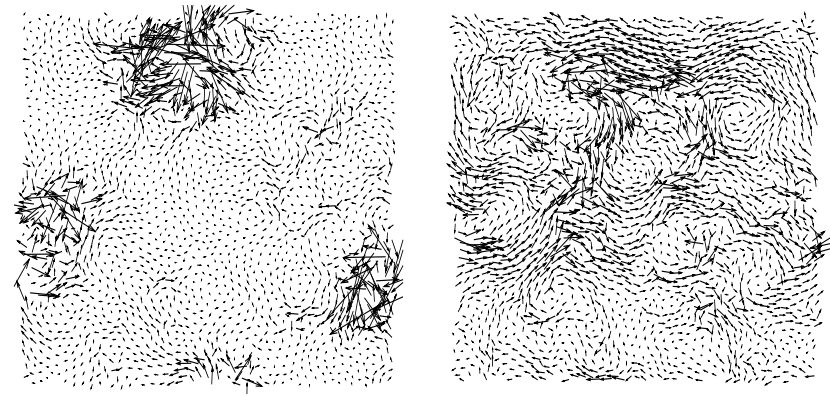

FIG. 1: Mismatch field $\left\{\mathbf{d}_{i}\right\}$ for the $2 \mathrm{D}$ soft spheres binary mixture of Ref. [16] at $T=1.0$ (left) and $T=0.46$ (right) for homogeneous shear deformation with amplitude $\gamma=0.001$. For better visibility, the lengths of the vectors are multiplied by a factor 14 and 600 for $T=1.0$ and $T=0.46$, respectively.

fluctuations of the system. At low temperatures, instead, $\left\{\mathbf{d}_{i}\right\}$ have much smaller magnitudes for the same $\gamma$ (see figure caption for details) but, at the same time, they appear rather spatially correlated in magnitude and in direction. These two kinds of behavior are ubiquitous in our numerical study and appear systematically for a wide range of $\gamma$ values [18]. Interestingly enough, in all cases considered, the transition from the one to the other behavior is coupled to the onset of the supercooled regime (for example, for the system $(c)$ in Ref. [16] the onset of the glassy dynamics can be set around $T \approx 0.46$ ). These findings support the idea that a qualitative change of the response of IS to deformation is strongly correlated to the onset of the cooperative dynamics [7].

A first, simple characterization is given in terms of the mean length of the mismatch vectors $l_{d}=$ $\left\langle N^{-1} \sum_{i} \mathbf{d}_{i}^{2}\right\rangle^{1 / 2}$. In Fig. 2, $l_{d}(\gamma, T) / l_{d}(\gamma, T=1)$, obtained in the system $(b)$ using the deformation $(i)$ of amplitude $\gamma$ and wave vector $k_{1}=\pi / L$, is plotted as a function of the temperature $T$. For sufficiently small values of $\gamma$, the relative global magnitude of the mismatch field shows a rather steep decrease with decreasing temperature. In fact in Ref. [7], it has also been observed that the critical deformation amplitude to cause IS transitions strongly depends on temperature. Remarkably, there are clearly two well distinguished temperature regimes for the typical length of the mismatch vectors. In addition, the transition from the high temperature to the low temperature one becomes steeper for small $\gamma$ and takes place at the onset of the cooperative dynamic regime $(T \simeq 0.4)$, i.e. very close to $T_{\mathrm{eIS}}$, where the IS energy starts to strongly depend on temperature, and well above structural arrest $\left(T_{\mathrm{MCT}} \simeq 0.303\right)[15,17$.

An estimate of the fraction of particles involved in the rearrangements described by the mismatch field can be obtained by the dimensionless width of the length distribution $\Pi=\left\langle N^{-1}\left[\Sigma_{i} \mathbf{d}_{i}^{2}\right]^{2} / \Sigma_{i}\left[\mathbf{d}_{i}^{2}\right]^{2}\right\rangle$ (the participation ratio of Ref. [20]). In the main frame of Fig. 3. $\Pi$ is plotted as a function of temperature $T$ for $\gamma=10^{-4}, 10^{-3}$ and $10^{-2}$ for the same system and the same wave vector 


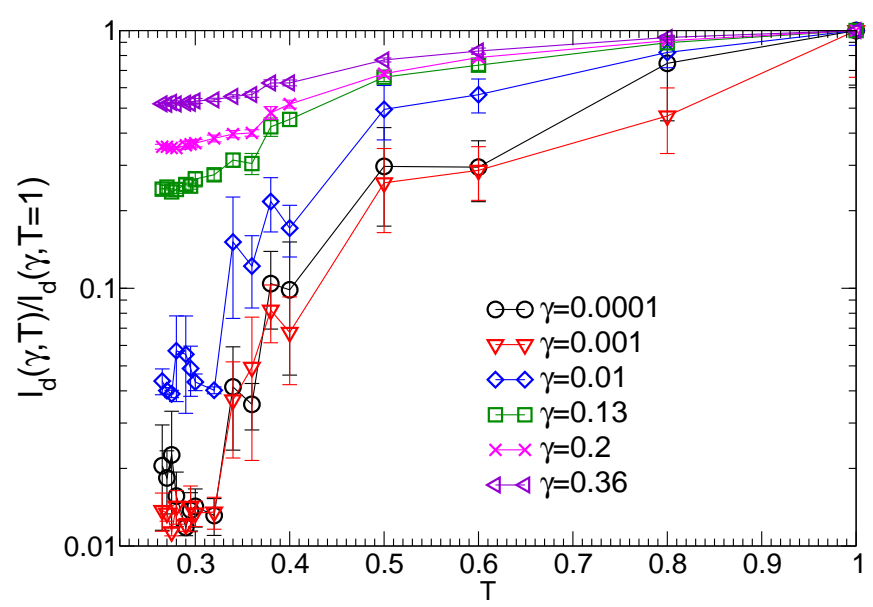

FIG. 2: (color online). Normalized mean length of mismatch field $l_{d}(\gamma, T) / l_{d}(\gamma, T=1)$ as a function of $T$ in the 3D binary mixture of Ref. [15], obtained with the spatially modulated deformation $(i)$ of amplitude $\gamma$ and wave vector $k_{1}$.

as in Fig. 2, The width $\Pi$ increases with decreasing temperature below $T=0.4$. In particular, for the very small values of $\gamma$ shown in the figure, $\Pi$ displays a rather sharp increase and shows that the fraction of particles involved in such non-affine deformation of the IS increases from $10 \%$ to $40 \%-60 \%$. In order to further characterize the nature of the non-affine rearrangements we have measured the average degree of correlation $C(r)$ between the directions of the mismatch vectors of different particles $i$ and $j$ separated by a distance $r, C(r)=\left\langle\mathbf{d}_{i} \cdot \mathbf{d}_{j}\right\rangle /\left\langle\mathbf{d}_{i}^{2}\right\rangle$. In the inset of Fig. 3. $C(r)$, obtained for $\gamma=0.01$ in the system $(b)$ with the deformation $(i)$, is plotted as a function of $r$. The data show that the low temperature regime is characterized by a definitely larger degree of correlation at the same distance $r$. Moreover, the distribution of values of $\mathbf{d}_{i} \cdot \mathbf{d}_{j} /\left\langle\mathbf{d}_{i}^{2}\right\rangle$ for pairs of particles separated by distance $r \pm d r$, plotted in Fig. 目, widens strongly at low temperature, indicating that the extended correlation in the response of IS to deformation corresponds to a high degree of heterogeneity. This feature is strikingly similar to what is observed in the dynamics with the average time correlation and the fluctuations around this average, generally used to quantify dynamical heterogeneities. But it is observed here, in the same range of temperatures [17], in a static quantity. On the whole, the emerging new scenario is that the onset of cooperativity in the real-time dynamics corresponds to growth of correlated and heterogeneous domains in the non-affine deformations of the IS.

The same qualitative behavior is obtained in the entire range $10^{-4} \leq \gamma \lesssim 0.1$ and in the systems $(a)$ and $(c)$ for both deformations $(i)$ and (ii). We have also found in all the systems considered that, when $\gamma \geq 1.0$, the mismatch field always closely resembles the one obtained at high temperature. This indicates that beyond $\gamma \simeq 1.0$

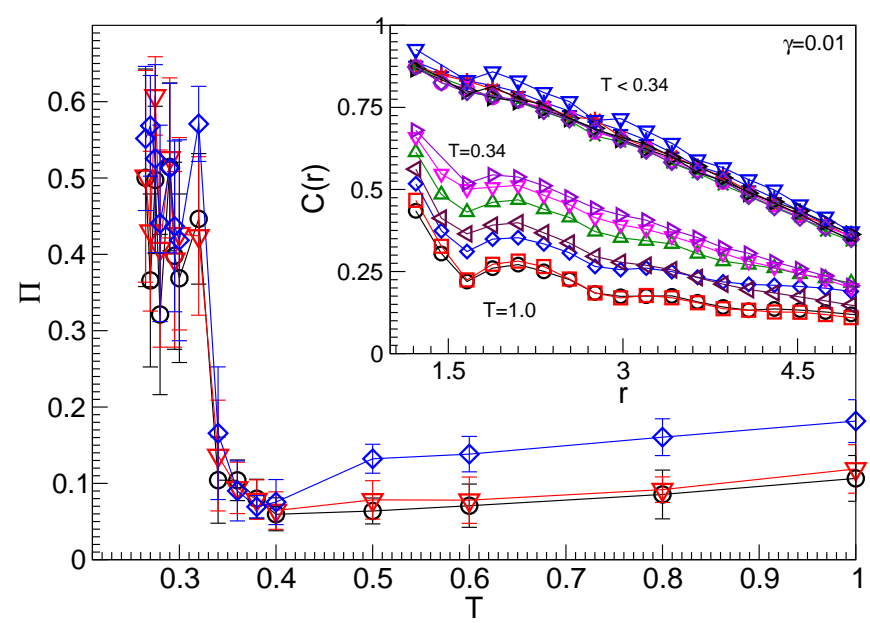

FIG. 3: (color online). Main frame: The participation ratio $\Pi$ as a function of the temperature for the 3D binary mixture of Ref. 15], in the case of the spatially modulated deformation (i) of amplitude $\gamma=10^{-4}, 10^{-3}, 10^{-2}$. Inset: Average degree of correlation $C(r)$ of the direction of the mismatch vectors separated by a distance $r$ for $\gamma=0.01$.

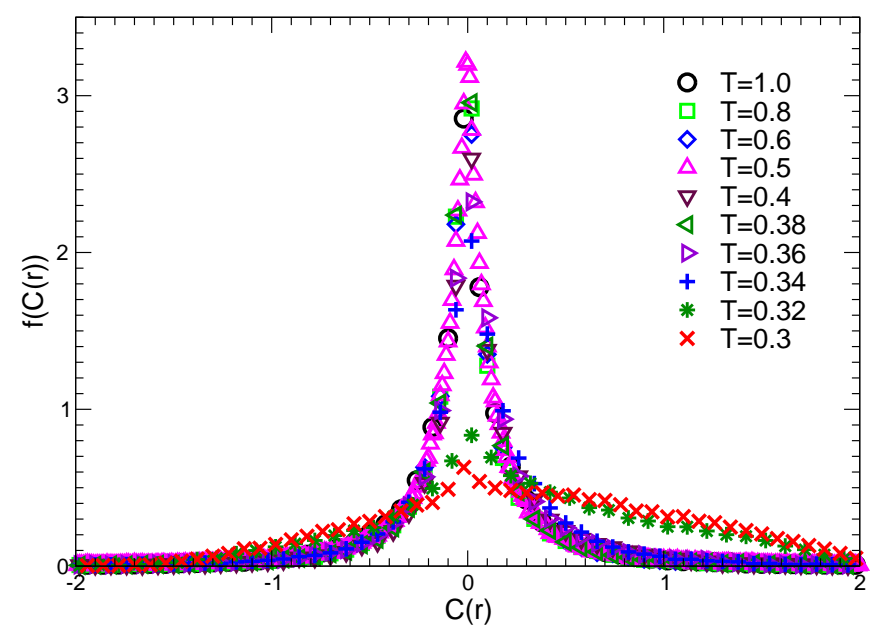

FIG. 4: (color online). Distributions of degree of correlation $C(r)$ between particles at a distance $r=5$ in particle diameter units. The same system and the same spatially modulated deformation ( $i$ ) as in Fig. 3 was chosen with deformation amplitude $\gamma=0.01$.

the amplitude of the deformation is sufficient to erase any distinctive feature of the low temperature structure of the IS, as in a sort of Lindemann criterion [ [ ] . Finally, the details of temperature dependence of the different quantities, e.g. the crossover width or the values of $\Pi$, depend to some extent on the specific system considered (e.g. the crossover is always smoother in the 2D system) and on the type of deformation [18]. 
Conclusions. The presented results reveal for the first time that the onset of the glassy dynamics corresponds to the onset of cooperative heterogeneous rearrangements in the response of IS to external, static deformations. In fact we have shown that in the liquid state, as a response to the external deformation, the IS displays large and uncorrelated rearrangements, dominated by thermal fluctuations. Near the onset of the cooperative dynamics, the response of the IS to small external deformation is instead characterized by small, collective non-affine rearrangements involving a relevant fraction of particles [13]. To our knowledge, this feature of the IS had not been recognized until now. It has been possible here, thanks to the procedure designed in Ref. [13] which strongly relies on the many-body nature of the IS construction. An interesting advantage of our approach is in avoiding calculations based on the dynamics, which usually require very long simulation times. The extent of such an advantage can be better quantified by systematically comparing our procedure to the dynamics [18].

The heterogeneous domains of the IS, determined by collective non-affine displacements, are strongly evocative of the cooperatively rearranging regions observed in the dynamics and are in fact detected in the same range of temperatures. We propose therefore that they are actually the IS counterpart of the cooperatively rearranging regions. If this is the case, they would deliver a static correlation length which grows significantly in the su- percooled regime, where the dynamic correlation length related to dynamic cooperativity starts to grow. From this point of view, this study suggests a new path for the investigations of structural signatures of glassy dynamics. A more sophisticated analysis of the non-affine displacement field, for example, adds useful insights in this direction [17]. Finally, our results have also interesting connections with the studies of elastic heterogeneities in amorphous solids [20, 21, 22, 23, 24, 25, 26, 27]. Their typical length scale has actually been related to the nonaffine part of the displacement field generated in response to an elastic deformation. In Ref. [26], where a quantitative estimate of this length scale is obtained in a realistic model of amorphous silica, the connection between the typical length scale of elastic heterogeneities in the amorphous solid and the length scale typical of dynamical heterogeneities (which is actually of comparable magnitude) in the supercooled liquid is suggested. Our results support this idea and offer a way to extend those studies to finite temperatures. On approaching the supercooled regime from the high temperature fluid, we find a signature of the onset of dynamic cooperativity in a purely static quantity: the non-affine, collective rearrangements of the IS show a striking similarity to cooperative, dynamical phenomena.

Acknowledgements. This project has been supported through EU-NSF contract NMP3-CT-2005-016375 of the European Community.
[1] G. Adam and J.H. Gibbs, J. Chem Phys. 43 (1965) 139; U. Pschorn et al., Macromolecules 24, 398 (1991); M.D. Ediger, Ann. Rev. Phys. Chem. 51 (2000) 99; E.V. Russel and N.E. Israeloff, Nature 408 (2000) 408; E.R. Weeks et al., Science 287 (2000) 627; L. Berthier et al., Science 310 (2005) 1797.

[2] S. Butler and P. Harrowell, J. Chem. Phys. 95 (1991) 4466; W. Kob et al., Phys. Rev. Lett. 79 (1997) 2827; S. Franz et al., Philos. Mag. B 79 (1999) 1827.

[3] X.Y. Xia and P.G. Wolynes, PNAS 97 (2000) 2990; J.P. Garrahan and D. Chandler, Phys. Rev. Lett. 89 (2002) 035704; E.J. Saltzman and K.S. Schweizer, J. Phys. Chem. B 108 (2004) 19729; G. Biroli and J.-P. Bouchaud, EPL 67 (2004) 21; G. Biroli et al. Phys. Rev. Lett. 97 (2006) 195701.

[4] K. Binder and W. Kob, Glassy Materials and Disordered Solids, World Scientific, 2005.

[5] A. Widmer-Cooper, P. Harrowell, and H. Fynewever, Phys. Rev. Lett. 93 (2004) 135701; A. Widmer-Cooper and P. Harrowell, Phys. Rev. Lett. 96 (2006) 185701.

[6] M. Goldstein, J. Chem. Phys. 51 (1969) 3728; F.H. Stillinger and T.A. Weber, Science 225 (1984) 983; S. Sastry, P.G. Debenedetti, and F.H. Stillinger, Nature 393 (1998) 554; K. Broderix et al., Phys. Rev. Lett. 85 (2000) 5360; E. La Nave, S. Mossa, and F. Sciortino, Phys. Rev. Lett. 88 (2002) 225701.

[7] S.S. Ashwin et al. J. Phys. Chem. B 108 (2004) 19703.

[8] G. Fabricius and D. Stariolo, Physica A, 331 (2004) 90.

[9] D. Coslovich and G. Pastore, EPL 75 (2006) 784.
[10] S. Capaccioli, G. Ruocco, and F. Zamponi, condmat/0710.1249.

[11] S. Mossa and G. Tarjus, J. Chem. Phys. 119 (2003) 8069; J. Non Cryst. Sol. 352 (2006) 4847.

[12] A. Cavagna, T.S. Grigera and P. Verrocchio, Phys. Rev. Lett. 98, 187801 (2007).

[13] H.C. Öttinger, Phys. Rev. E 74 (2006) 011113.

[14] W. Kob and H.C. Andersen, Phys. Rev. Lett. 73 (1994) 1376.

[15] H.M. Carruzzo and C.C. Yu, Phys. Rev. E 66 (2002) 021204; Q. Yan, T.S. Jain, and J.J. de Pablo, Phys. Rev. Lett. 92 (2004) 235701.

[16] D.N. Perrera and P. Harrowell, J. Chem. Phys. 111 (1999) 5441.

[17] See auxiliary material in the Appendixes.

[18] P. Ilg, E. Del Gado, M. Kröger and H.C. Öttinger, in preparation.

[19] S.J. Plimpton, J. Comp. Phys. 117 (1995) 1.

[20] F. Leonforte et al., Phys. Rev. B 72 (2005) 224206.

[21] D.N. Theodorou and U.W. Suter, Macromolecules 19 (1986) 379.

[22] D.L. Malandro and D.J. Lacks, J. Chem. Phys. 110 (1999) 4593.

[23] J.S. Langer, Phys. Rev. E 70 (2004) 041502.

[24] G. Picard et al., Phys. Rev. E 71 (2005) 010501(R).

[25] C.E. Maloney and A. Lemaître, Phys. Rev. Lett. 93 (2004) 195501; Phys. Rev. E 74 (2006) 016118.

[26] F. Leonforte et al., Phys. Rev. Lett. 97 (2006) 055501.

[27] G.J. Papakonstantopoulos et al., Phys. Rev. E 77 (2008) 
041502 


\section{APPENDIX A: DETAILS OF NUMERICAL SIMULATIONS}

For the two 3D systems $(a)$ and (b), we have performed NVE and NVT molecular dynamics simulations of systems containing $N=2048$ particles. For system $(a)$ and (b), the chosen number densities and corresponding size of the simulation box are $\rho=1.2, L=11.95$ [A1] and $\rho=0.6, L=15.06$ [A2], respectively. For the 2D system $(c)$, we have performed constrained molecular dynamics simulations in the NPT-ensemble (with $N=500,2048$, and 8000), with Nosé-Hoover thermo- and barostats ensuring on average a fixed temperature $(0.3 \leq T \leq 2)$ and pressure $(P=13.5)$ value [A3].

\section{APPENDIX B: THE MISMATCH DISTRIBUTION}

The motivation for studying the mismatch field is the idea, presented within the thermodynamic modeling approach of Ref. A5], that the behavior of the system under deformation can be described using the underlying inherent structure. Since we here study the liquid regime, we have chosen the mismatch field $\left\{\mathbf{d}=X^{\mathrm{dq}}-X^{\mathrm{qd}}\right\}$ in order to subtract the affine part of the deformation, such that d only accounts for non-affine contributions to the deformation. For a crystalline solid, instead, the mismatch has to be more conveniently considered with respect to $X^{\mathrm{q}}$, i.e. the IS of the original configuration. In this case, $\mathbf{d}$ is therefore a measure of the difference between two configurations of the solid.

There are number of related and special cases which help to clarify the meaning of the mismatch vectors $\left\{\mathbf{d}_{j}\right\}$, or equally, the mismatch vector field $\mathbf{d}(\mathbf{r})$ : (A) For the special case of a perfectly flat energy landscape, $\mathbf{d}$ vanishes identically; (B) In a real fluid, the mismatch vector field $\mathbf{d}$ does not vanish even at high temperatures, in fact its magnitude tends to become large in that case, but it will be orientationally correlated only over short distances and vanish when averaged over volume elements of a few particle diameters; (C) In the case of extremely small deformations that do not allow to overcome even the slightest energy barriers, $\mathbf{d}(\mathbf{r})$ is identical to minus the imposed deformation at location $\mathbf{r}$. This limit has not been reached for the range of deformation amplitudes $\gamma$ that we have considered (see also next section); (D) Applying rather large deformations $\gamma \gtrsim 10^{-1}$ within our procedure (but out of focus of the present study) gives information about sizes of inherent structure basins, similar to the work of [A6]; (E) For very low temperatures, where the initial configuration is an amorphous solid, $X \rightarrow X^{\mathrm{q}}$, the mismatch field coincides with the quantity studied in Ref. [A7], where it was used in order to extract the non-affine displacements in amorphous solids.

From the measured mismatch fields $\left\{\mathbf{d}_{j}\right\}$ we have further compiled ensemble averaged probability distributions of mismatch strength; denoted as $h(d)$, where $d$ is the norm of the mismatch vector. For case (C) con-

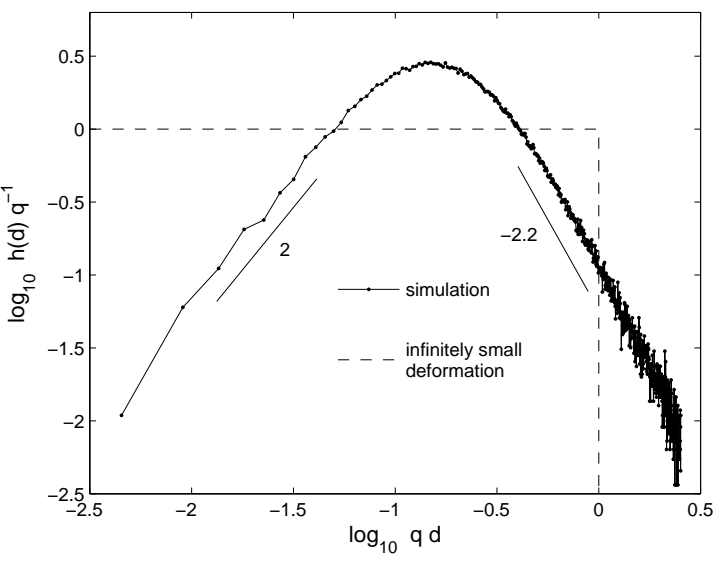

FIG. 5: Scaled probability distribution function of mismatch strengths, with $q \equiv 2 /(\gamma L)$, for $T=0.46$ and $\gamma=10^{-3}$ for system (c). Also shown is the reference distribution one would observe for extremely small deformations, as described in the text part.

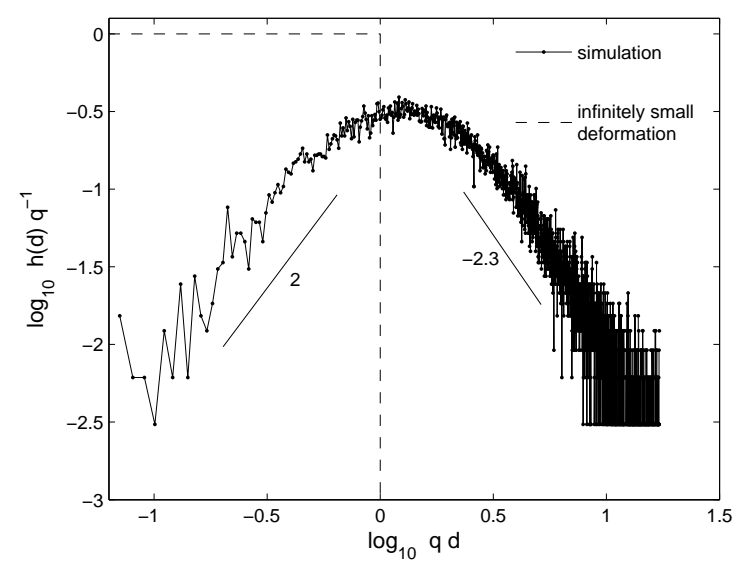

FIG. 6: Same as Fig. 5 for $T=0.8$ and $\gamma=10^{-3}$.

sidered above, the distribution is flat and vanishes for $d \geq \gamma L / 2$ (for the case of homogeneous shear strain applied of size $\gamma$ to a homogeneous system of linear size $L)$. In Figs. 5 and 6 we quantitatively demonstrate for two representative temperatures and strain amplitudes $\gamma$, that even for deformations as small as those required for the approach presented in the manuscript, this regime is not observed. Moreover, the figures show that the distributions are characterized by an 'initial' (at small $\gamma$ ) parabolic increase with $d$, a 'later' decrease well described by a power law, and a finite range of displacement strengths which ensures integrability and calculation of the moments $\Pi$ and $l_{d}$ presented in Figs. 2 and 3 of the manuscript. The occurrence of a significant fraction of values of $q d$ between 1 and 10 in Fig. 6 underlines the enormous freedom of rearranging individual particles in the liquid state.

The strong discrepancy between measured distribu- 
tions and the reference distribution, as shown in Figs. 5 and 6 for two different temperatures, sheds some light on the fact that a picture of energy valleys being large enough to accommodate small deformations without affecting the inherent structure begins to fail at deformations small compared to the ones needed to recognize the occurrence of the glass transition.

\section{APPENDIX C: PROPENSITY OF PARTICLES TO MOTION}

For the model of Ref. [A2], to which most of the data shown in the paper refer, no study of dynamical heterogeneity was available in the literature. We have calculated the local propensity to motion $p$ as defined in Ref. [A9] from the distribution of particle displacements after a time interval $\simeq 1.5 \tau_{e}$, where $\tau_{e}$ is the time at which the intermediate scattering funcion, measured at the wave vector of the first peak in the structure factor, has decayed to $1 / e$. The distribution is calculated from $N_{r}=20$ separate simulation runs over a fixed time interval, all starting from the same particle configuration but with momenta randomly assigned from the MaxwellBoltzmann distribution at a certain temperature. In Ref. A9] it is shown that the probability distribution $f(p)$ becomes asymmetric and much wider at the onset of the supercooled regime. In Fig. 7 we have plotted the distribution of propensity values at various temperatures: the data show the change in the shape of the distribution at temperatures below $T=0.4$ and higher than $T_{\mathrm{MCT}}$. The distribution of degree of spatial correlation plotted in Fig. 4 of the manuscript displays a strikingly similar qualitative change in the same range of temperature.

\section{APPENDIX D: SEARCH FOR CORRELATIONS BETWEEN DISTINCT APPROACHES}

One of the possible developments of this work is in the direction of quantitatively characterizing correlations between the cooperative domains observed in the response of IS and the heterogeneities observed in particle dynamics. In principle, this would require a systematic analysis of the different measures of dynamical heterogeneities proposed in the literature A8], but, to get started, let us consider the local propensity to motion $p$ discussed in the previous secion. A significant change of shape is also observed for the distribution $h(d)$ of magnitudes of the mismatch vectors, as discussed in the previous section, and even more strikingly for the distribution of $\left\langle\mathbf{d}_{i} \cdot \mathbf{d}_{j}\right\rangle, f(C)$, see Fig. 4 of the manuscript. Starting from these first indications, we have calculated conditional probability distributions for the propensity and mismatch lengths. We have also evaluated, at different temperatures, different correlation coefficients between propensity and mismatch length distributions, the latter calculated for different values of deformation amplitudes. We can rule

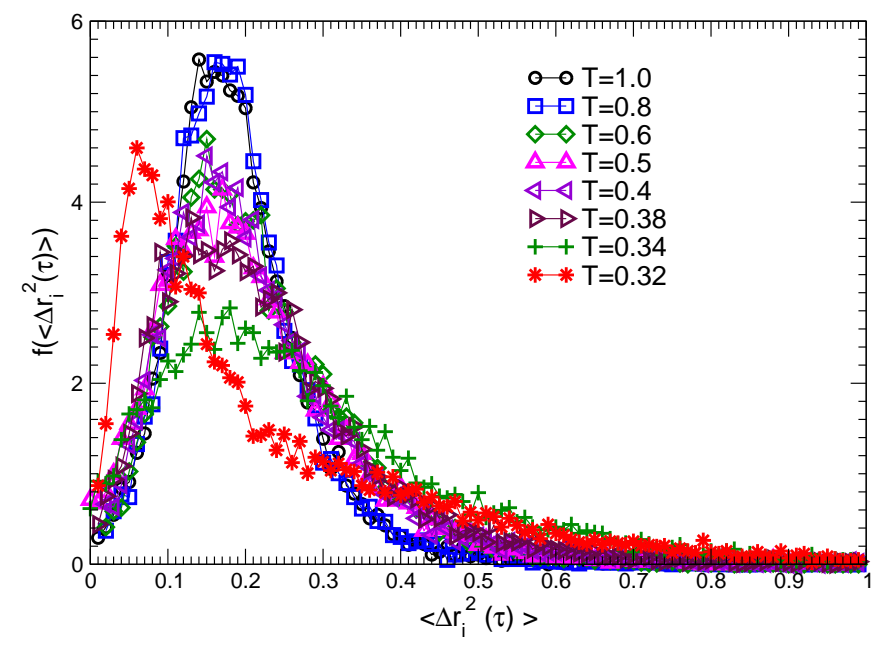

FIG. 7: Distribution of particle displacements (propensity to motion) at time $\tau \simeq 1.5 \tau_{e}$ at different temperatures.

out a clear signal of a simple and straightforward correlation between $\left|\mathbf{d}_{j}\right|$ and $p_{j}$. This is illustrated e.g. in Fig. 8, where the propensities and mismatch vectors are visualized on top of the particle configuration for the $2 \mathrm{D}$ system $(c)$. Correlations between particle propensity and the spatial correlation of the mismatch vectors give instead more encouraging indications, as also suggested by the cooperative nature of the mismatch vectors at low temperature. By defining the coarse-grained mismatch field $D(r)=\left\langle\mathbf{d}^{2}(r)\right\rangle_{j}$, where $\langle\ldots\rangle_{j}$ indicates the average over the $N_{j}$ particles contained in the volume $V_{j}$ of linear size $r$ [A], and the analogue coarse-grained propensity field $P(r)$, we have calculated their spatial correlation $\langle D(r) P(r)\rangle$, plotted in Fig. 8 for the model system (b). Such correlations apparently become longer ranged at low temperatures. The same behavior is observed in different model systems, and for different shear amplitudes.
[A1] W. Kob and H. C. Andersen, Phys. Rev. Lett. 73 (1994) 1376.
[A2] H. M. Carruzzo and C. C. Yu, Phys. Rev. E 66 (2002) 021204. 

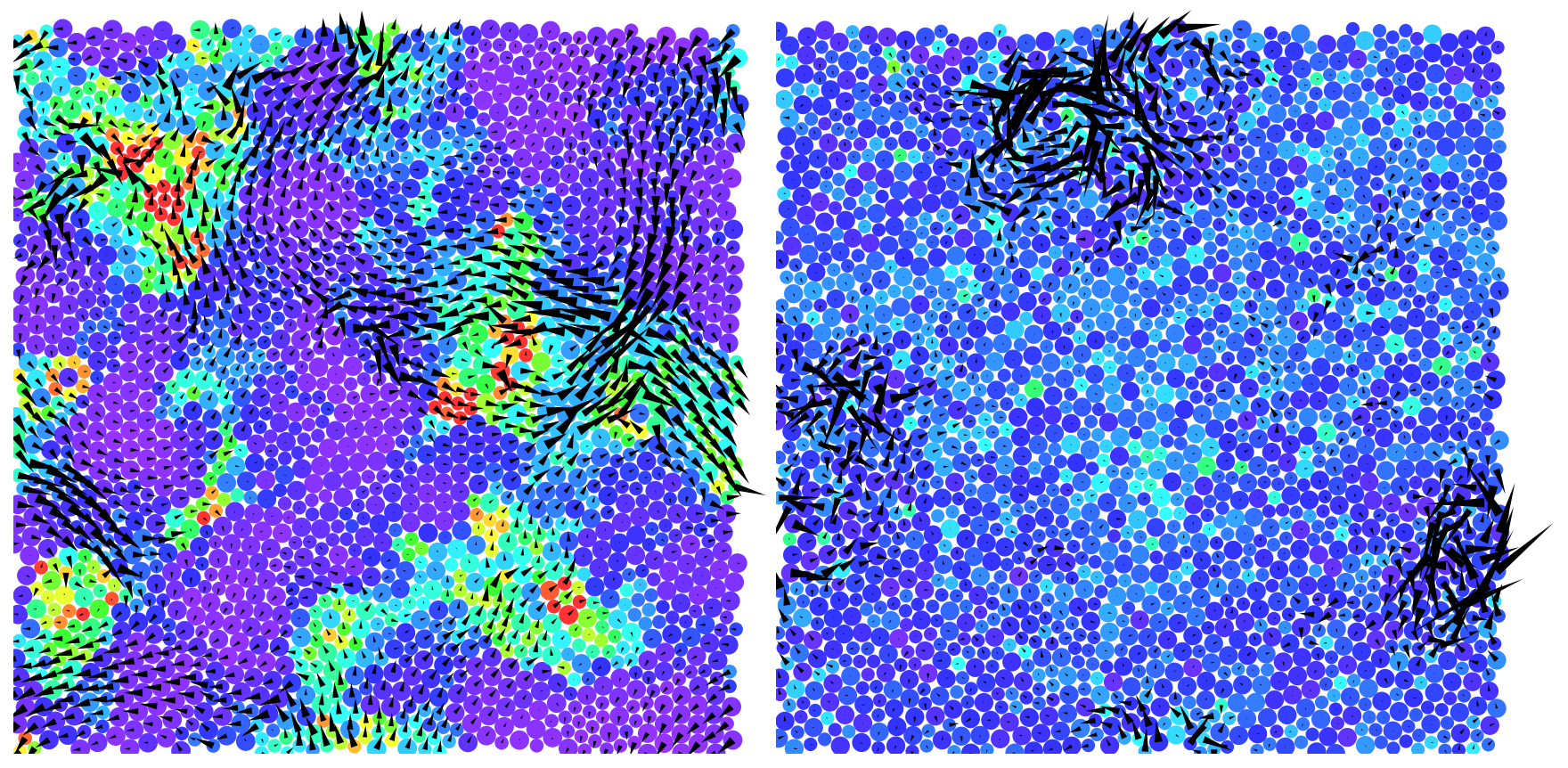

FIG. 8: Visualization of particle propensity and mismatch vectors for the system $(c)$. Increasing propensity is color coded from violet (dark) to red (bright). Same conditions are chosen as in Fig. 1 of the manuscript with $T=0.4$ (left) and $T=1.0$ (right).

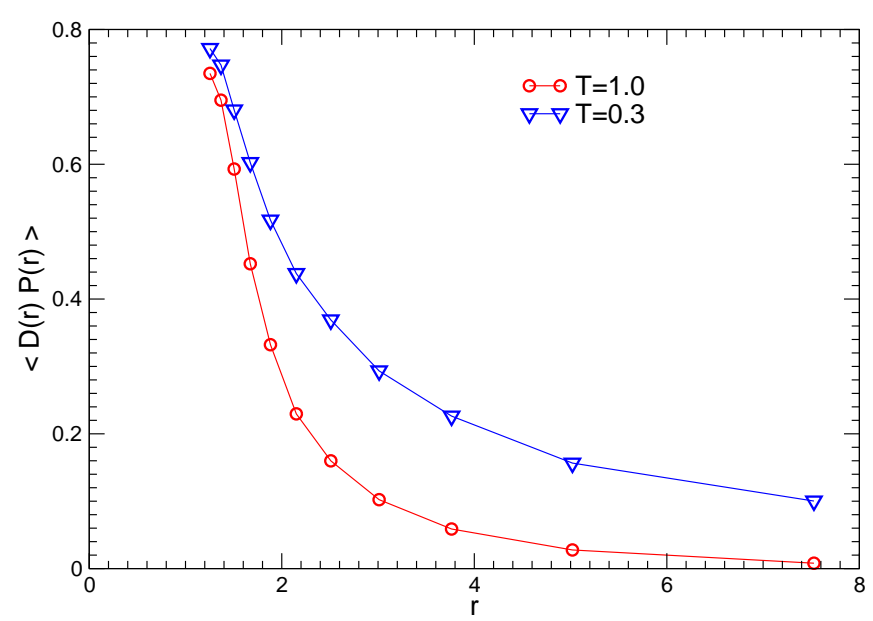

FIG. 9: Spatial correlations between the coarse grained fields $D(r)$ and $P(r)$ as a function of the coarse graining distance $r$.
[A3] D. N. Perrera and P. Harrowell, J. Chem. Phys. 111 (1999) 5441.

[A4] P. Ilg, E. Del Gado, M. Kröger and H. C. Öttinger, in preparation.

[A5] H. C. Öttinger, Phys. Rev. E 74 (2006) 011113.

[A6] S. S. Ashwin, Y. Brumer, D. R. Reichman and S. Sastry, J. Phys. Chem. B 108 (2004) 19703.

[A7] F. Leonforte, R. Boissière, A. Tanguy, J. P. Wittmer, and J.-L. Barrat. Phys. Rev. B 72 (2005) 224206.

[A8] W. Kob, C. Donati, S.J. Plimpton, P.H. Poole, and S.C. Glotzer, Phys. Rev. Lett. 79 (1997) 2827; S. Franz, C. Donati, G. Parisi and S.C. Glotzer, Philos. Mag.B 79 (1999) 1827; Lacevic et al, J. Chem. Phys.119, 7386 (2003).

[A9] A. Widmer-Cooper, P. Harrowell and H. Fynewever, Phys. Rev. Lett. 93 (2004) 135701. 